\title{
Performance test of a triple GEM detector at CERN n_TOF facility
}

\author{
G. Claps, G. Croci, F. Murtas, A. Pietropaolo, S. Puddu, C.T. Severino, M. Silari
}

\begin{abstract}
The application of a triple GEM (Gas Electron Multiplier) for neutron detection was tested at the n_TOF facility at CERN. n_TOF allows the neutron energy distribution to be measured via a $\sim 185 \mathrm{~m}$ time of flight path. A $20 \mathrm{GeV} / \mathrm{c}$ proton beam hits a lead target generating a neutron spectrum ranging from thermal energies to the $\mathrm{GeV}$ region. Due to their long flight path and short proton pulse, the neutron arrival times at the experimental area define their energy [1]. A triple GEM detector with a $60 \mu \mathrm{m}$ Polyethylene (PE) neutron converter and $40 \mu \mathrm{m}$ of Aluminium, filled with an $\mathrm{Ar}-\mathrm{CO}_{2} \mathbf{7 0 - 3 0 \%}$ mixture, was installed a few meters downstream of the experimental area, just in front of the beam dump. The measurements were purely "parasitic"; they were conducted in parallel and without interfering with the official n_TOF scientific program. Using the n_TOF trigger it is possible to synchronize the GEM data acquisition in order to select a given neutron energy window and measure the detector efficiency as a function of neutron energy. Changing the detector gain, it is possible to perform these measurements with a low $\gamma$ background level. Thanks to the pixelated read-out the neutron beam spot and the efficiency of the detector have been measured.
\end{abstract}

\section{INTRODUCTION}

For the first time the performance of a triple GEM detector,

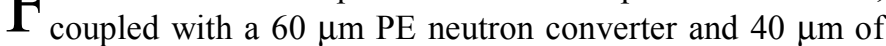
Aluminium, was tested at the $\mathrm{n}$ TOF facility at CERN, to examine possible future applications in neutron dosimetry and neutron beam monitoring.

The $\mathrm{n}$ TOF [1] facility allows the neutron energy distribution to be measured via a $\sim 185 \mathrm{~m}$ time of flight path. A proton beam with momentum of $20 \mathrm{GeV} / \mathrm{c}$ from the Proton Synchrotron (PS) hits a lead target. The produced neutrons are moderated with borated water, in order to introduce a cut in the thermal region [2].

\footnotetext{
Manuscript received November 16, 2012. (Write the date on which you submitted your paper.) This work was supported in part by the CERN , ARDENT Marie Curie Program, LNF-INFN, LHEP Bern University and CNR-IFP.

The authors wish to thank E. Berthoumieux, E. Chiaveri and C. Guerrero Sanchez of the n_TOF collaboration for their scientific and technical support.

S. Puddu (tel: +41227673535, e-mail: silvia.puddu@ern.ch) and C. T. Severino are with CERN, 1211 Geneva 23, Switzerland and Universtity of Bern, LHEP, Sidlerstrasse 5, 3012 Bern, Switzerland

F. Murtas is with CERN and LNF-INFN via Fermi 40, 00044 Frascati (Roma) Italy

M. Silari is with CERN

A. Pietropaolo and G. Croci are with CNR-IFP, via R. Cozzi 53, 20125 Milano, Italy

G. Claps is with LNF-INFN
}

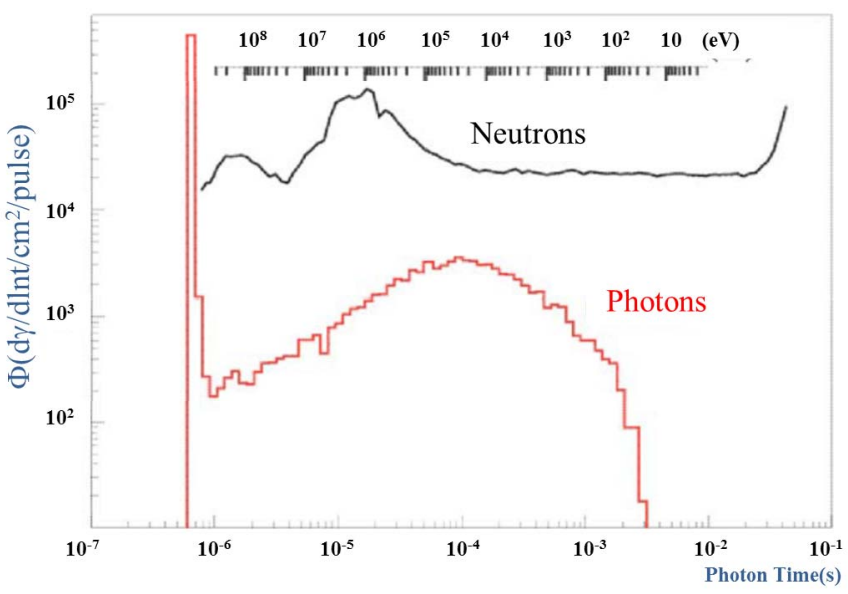

Fig. 1. $\mathrm{n}_{-}$TOF neutron spectrum and $\gamma$-background from FLUKA simulations [1]

A neutron energy spectrum is obtained from thermal energies to the $\mathrm{GeV}$ region, Fig. 1 .

\section{EXPERIMENTAL SET-UP}

The detector is a prototype optimized for neutron measurements. A triple GEM [3], [4] chamber with a specific fast neutron cathode converter has been used.

As shown in Fig. 2 neutrons are converted into proton in PE thanks to the (n, p) reaction. To ensure direction capability protons that come from neutrons with too wide an angle with respect to the normal of the converter are stopped in the $\mathrm{Al}$ layer [5].

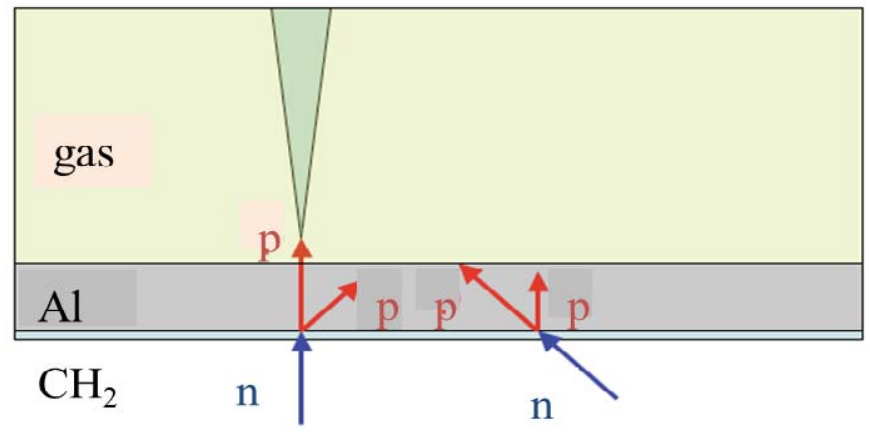

Fig. 2. Coverter and drift zone of triple GEM detector for fast neutrons

The triple GEM is filled with an $\mathrm{Ar}-\mathrm{CO}_{2} 70-30 \%$ gas mixture; the anode has $1286 \times 12 \mathrm{~mm}^{2}$ pads organized in an $8 \times 16$ matrix, for a total of $10 \times 10 \mathrm{~cm}^{2}$ sensitive surface. The 
data acquisition (Fig. 3) is made via a FPGA-based motherboard [6], that can be externally triggered and can also measure the hit rate for each channel.

Several measurements were made in order to evaluate the $\gamma$ background, the mean efficiency and the efficiency in function of the incoming neutron energy.

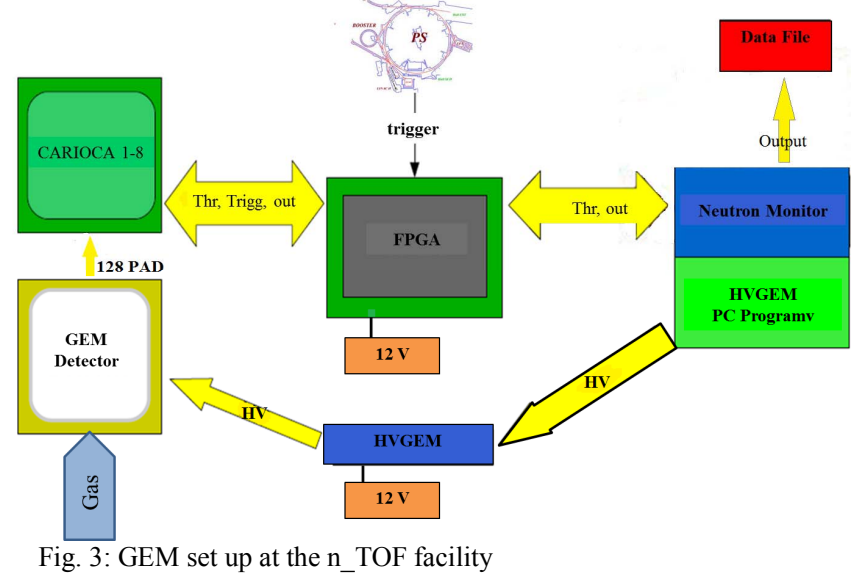

All measurements were carried out in "parasitic" mode over a period of 3 weeks. The detector was installed between the end of the beam pipe and the beam dump (Fig. 4); $200 \mathrm{~m}$ from the spallation target.

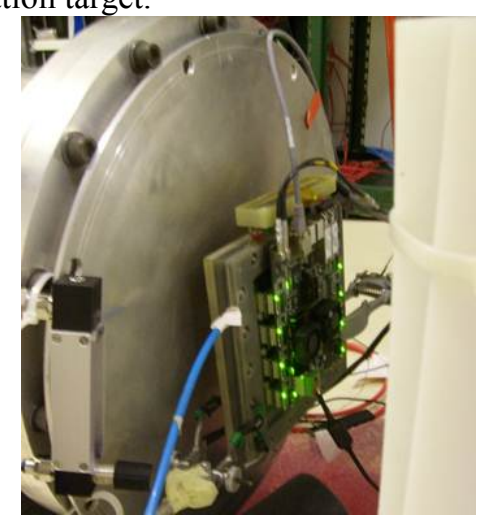

Fig. 4: the GEM installed between the beam pipe and the $\mathrm{n}_{-}$TOF dump

\section{REJECTION OF GAMMA BACKGROUND}

The dominant contributions to the $\gamma$-background (Fig. 1), come from reactions at the spallation target and are composed of the " $\gamma$-flash" from prompt $\gamma$-rays and a "slow" component from other reactions such as neutron capture [1]. Due to the proximity to the beam dump there is a secondary contribution from $\gamma$-rays due to neutron capture. However, thanks to the possibility to operate the GEM with low gain, it was possible to perform the measurements with the $\gamma$ background heavily reduced. In Fig. 5 a GEM gain scan during an n_TOF run and a similar scan with a ${ }^{137} \mathrm{Cs}$ source are compared, the $\gamma$-energy emission of which is similar to the n_TOF $\gamma$-flash [1].

For this proposal the GEM was randomly triggered with a $1 \mathrm{~s}$ gate. The insensitivity to the $\gamma$-background was assessed with a measurement triggering the acquisition in the neutron thermal region (where $(n, p)$ reactions do not occur in the PE converter and the signal in the GEM would only be due to the $\gamma$-background). To have a high rejection factor a working point of $870 \mathrm{~V}$ has been chosen.

HV scan with n TOF and Cs1.37 with a gate of 1 second

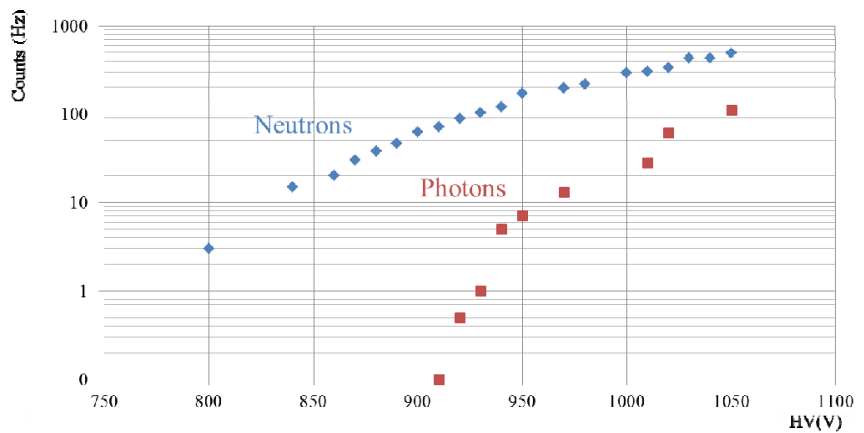

Fig. 5: Gain scan for $n_{-}$TOF and a Cs-137 source

\section{MEAN EFFICIENCY AND BEAM PROFILE}

Two kind of PS beams arrive at the spallation target, that are different essentially for the proton intensity. Thanks to this peculiarity, correlation and then the mean efficiency were measured (Fig. 6).

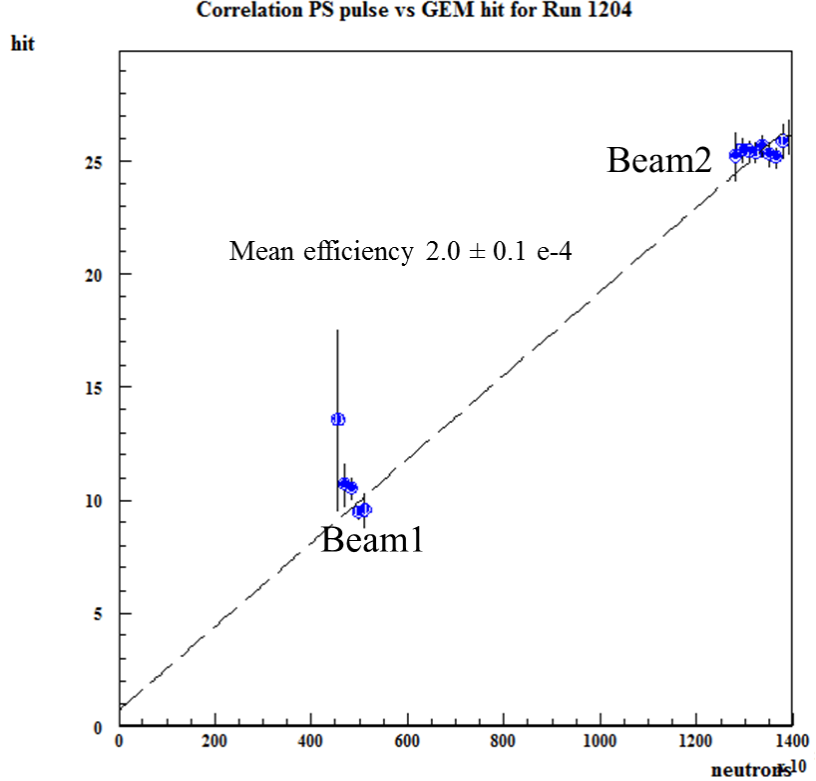

Fig. 6: correlation and efficiency

With a trigger delay of $2 \mu$ s, to avoid $\gamma$-flash and a gate of $10 \mathrm{~ms}$ the $2 \mathrm{D}$ beam spot was measured as shown in fig. 7 .
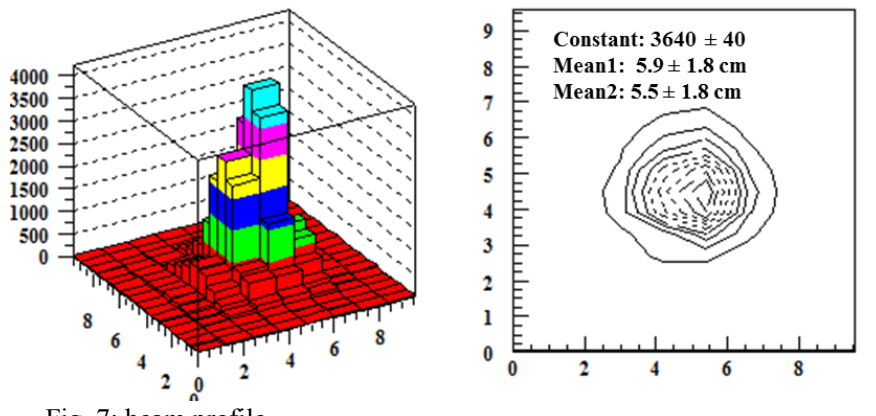

Fig. 7: beam profile 


\section{NEUTRON ENERGY SCAN}

Thanks to the FPGA software, delays from the PS trigger can be set ranging from $4.7 \mu$ s up to $63.7 \mu$ s in order to select the TOF (i.e. the energy) of the detected neutrons. The acquisition gate was changed from $100 \mathrm{~ns}$ up to $5 \mu$ s. Thanks to this procedure the efficiency in function of incoming neutron energy can be measured. In fig. 8 the comparison between neutrons that arrive at the detector and GEM counts is shown.
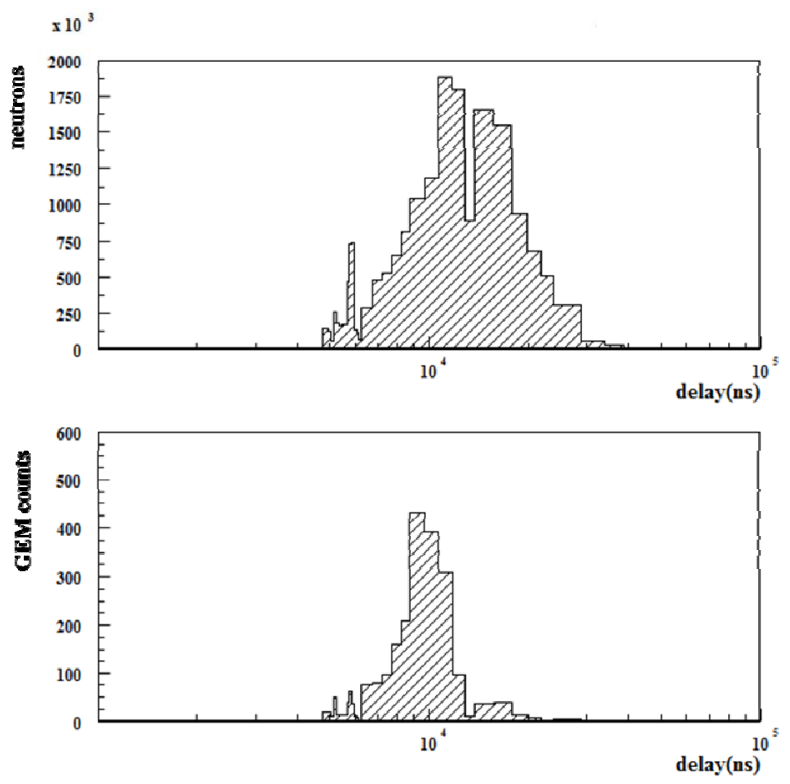

Fig. 8: Comparison between estimated neutron at the detector and GEM counts

The efficiency in function of neutron energy is shown in Fig. 9. Errors was calculated to be between $0.1 \%$ and $1 \%$, large oscillations in the first part of the spectrum need to be investigated because of the poor statistics in this region.

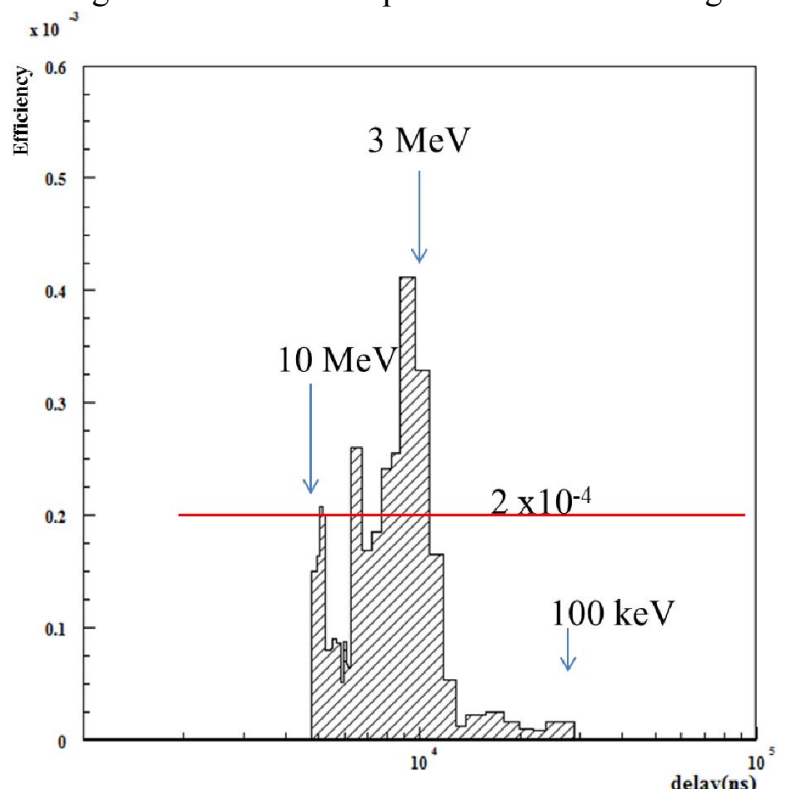

Fig. 9: GEM efficiency VS neutron TOF compared with mean efficiency

\section{CONCLUSION}

A triple GEM detector coupled with a fast neutron converter was tested at $n \_$TOF facility at CERN, showing a high $\gamma$ rejection. The response of the detector is linear and the mean efficiency was measured as $2 \times 10^{-4}$. The $2 \mathrm{D}$ beam spot is well recognized. Thanks to the FPGA-based acquisition, the behavior of the detector as a function of incoming neutron energy was measured.

\section{REFERENCES}

[1] E. Chiaveri et al, CERN n_TOF facility performance report, CERN-SL2002-053 ECT (2002).

[2] C. Guerrero Sanchez, The neutron beam and the associated physics program of the CERN n_Tof facility, ATS seminar

[3] F. Sauli NIM A386 531

[4] M. Alfonsi et al., The triple-Gem detector for the M1R1 muon station at LHCb, N14-182, 2005 IEEE-NSS

[5] F. Murtas et al., Triple GEM gas detectors as real time fast neutron beam monitors for spallation neutron sources, JINST_014P_0512

[6] Developed by A.Balla and G. Corradi and Electronic Group LNF-INFN 